\title{
Myocardial contractility recovery following acute pressure unloading after transcatheter aortic valve intervention (TAVI) in patients with severe aortic stenosis and different left ventricular geometry: a multilayer longitudinal strain echocardiographicanalysis
}

\author{
Sara Cimino ${ }^{1}$ - Sara Monosilio ${ }^{1} \cdot$ Federico Luongo $^{1} \cdot$ Matteo Neccia $^{1} \cdot$ Lucia llaria Birtolo $^{1} \cdot$ Nicolò Salvi $^{1}$. \\ Domenico Filomena $^{1} \cdot$ Massimo Mancone $^{1} \cdot$ Francesco Fedele $^{1} \cdot$ Luciano Agati $^{1} \cdot$ Viviana Maestrini $^{1}$
}

Received: 3 September 2020 / Accepted: 13 October 2020 / Published online: 30 November 2020

(C) The Author(s) 2020

\begin{abstract}
Aim of the present study was to describe the left ventricular longitudinal strain (LS) in all myocardial layers in patients with severe aortic stenosis (AS), preserved left ventricular ejection fraction (LVEF) in different LV geometry and to compare LS analysis before and early after acute LV unloading provided by transcatheter aortic valve implantation (TAVI). 68 patients were enrolled. LS was measured from the endocardial layer (Endo-LS), epicardial layer (Epi-LS) and full thickness of myocardium (Transmural-LS) before and after TAVI. Patients were divided in two groups accordingly with relative wall thickness (RWT): concentric LV hypertrophy (cLVH) vs eccentric LV hypertrophy (eLVH). Less impaired values of LS at baseline were observed, in all layers, in patients with cLVHas compared to patients with eLVH (Endo-LS was $-13.2 \pm 2$ vs $-11.1 \pm 3 \%, \mathrm{p}=0.041$; Epi-LS was $-11.8 \pm 1.8$ vs $-9.9 \pm 3 \%, \mathrm{p}=0.043$; Transmural-LS was $-12.3 \pm 1.8$ vs -10.49 $\pm 3.3 \%, \mathrm{p}=0.02$, respectively). A significant improvement in endocardial LS (Endo-LS) after TAVI was detected only in $\operatorname{cLVH}(-13 \pm 2$ vs $-14 \pm 2, p=0.011)$. Our findings documented that concentric LVH had better basal strain function and showed a better myocardial recovery after TAVI compared to eLVH.
\end{abstract}

Keywords Strain echocardiography · Aortic stenosis · TAVI

\section{Background}

In patients with aortic stenosis (AS), left ventricular hypertrophy (LVH) is considered to be an adaptive response to the increased afterload [1, 2]. LVH reduces systolic wall stress and helps to preserve LV ejection fraction (LVEF), but it may lead to some long term adverse consequences such as myocardial fibrosis, diastolic filling impairment and finally LV dysfunction [3]. Different types of LVH have been previously described. Gaasch and Zile [4], identified four groups of LV geometry, based on the estimation of LV mass and LV relative wall thickness (RWT). LVH by echocardiography

Sara Cimino

sara.cimino@uniroma1.it

1 Department of Cardiovascular, Respiratory, Nephrological, Anesthesiological and Geriatric, Science "Sapienza" University of Rome, Policlinico Umberto I, Rome Viale del Policlinico 155, 00161 Roma, Italy was defined as an increase of the LV mass index $>95 \mathrm{~g} / \mathrm{m}^{2}$ in women and $>115 \mathrm{~g} / \mathrm{m}^{2}$ in men. Concentric LVH $(\mathrm{cLVH})$ was characterized by a RWT $\geq 0.42$, while eccentric LVH $(\mathrm{eLVH})$ by a RWT $<0.42$. These myocardial changes are progressive and associated with symptoms' onset and prognosis worsening. More severe LVH and, specifically, eLVH pattern seems to be associated with poor prognosis after aortic valve replacement (AVR) [5]. Current guidelines recommend aortic valve replacement for patients with preserved LVEF only at symptoms' onset, remaining at this stage the main determinant of poor prognosis [6]. However, LVEF has several well know limitation. Longitudinal global strain (LGS) is more sensible to assess subclinical changes in LV function even in patients with preserved LVEF [7]. More recently, multilayer longitudinal strain (LS) has emerged as an innovative tool for a more detailed evaluation of LV mechanics providing a separate analysis in different myocardial layers. This is particularly useful in cardiac disease with expected involvement of sub-endocardial layer (significant 
coronary artery disease or pressure overload) $[4,8]$. Multilayer strain analysis in AS revealed that LS may be impaired in all myocardial layers (sub-endocardial, sub-epicardial and transmural), independently from LV shape [9]. Transcatheter aortic valve implantation (TAVI) is an effective therapeutic option for severe AS, with a significant prognostic impact and an association with cardiac performance improvement over time [10]. However, acute myocardial contractility response after afterload removal in different LV geometry has been not exhaustively described.

Aim of the present study was to examine the acute effect of TAVI in terms of pressure unloading, on LV mechanics using multilayer LS by 2D speckle-tracking echocardiography (ST-E) in different patterns of LV geometry to better understand the subset of AS patients that have potentially more benefits from LV unloading.

\section{Methods}

We enrolled 68 patients (mean age $82 \pm 5$ years, $34 \%$ male, $43 \%$ with previous history of coronary artery disease) with severe symptomatic AS and preserved LVEF, scheduled for TAVI. Inclusion criteria were: (a) severe AS defined by an aortic valve area $\leq 0.6 \mathrm{~cm}^{2} / \mathrm{m}^{2}$ by echocardiography, accordingly with current guidelines [6], (b) preserved LVEF ( $\geq 50 \%$ as calculated by 3D echocardiography (Dinamic Heart Model by Philips). Considering these parameters, patients with paradoxical low-flow low-gradient (PLFLG) AS were included.

All patients underwent 2D and 3D echocardiography at baseline and $5 \pm 2$ days after TAVI. LS by ST-E was measured from the endocardial layer (Endo-LS), epicardial layer (Epi-LS) and full thickness of myocardium (Transmural-LS) before and after the procedure. ST-E analysis was performed with QLab software v.13 by Philips. Analysis included the collection of clinical characteristics and 2D/3D echocardiographic features, such as LV volumes indexed for body surface area (BSA), and ejection fraction (LVEF), LV mass, $\mathrm{LV}$ diastolic function, right ventricular (RV) dimension and function and RV-LS. Patients with significant aortic regurgitation or mitral valve disease, pace-maker (PMK) rhythm and/or left bundle branch block (LBBB), unstable coronary artery disease (CAD) were excluded to avoid confounders. Patients with poor acoustic window and uncontrolled heart rate were excluded as contraindications to perform ST analysis. All patients fulfilled LVH hypertrophy criteria by LV mass index. Patients were divided in two groups, accordingly to RWT measurement. cLVH was more represented than eLVH in our study population (46 patients, $68 \%$, vs. 22 patients, $32 \%$ ). All clinical and demographic characteristics were collected, including stable CAD. The Ethic committee of "Policlinico Umberto I Hospital- Sapienza, University of Rome" approved the present study, and all patients provided written informed consent. Comprehensive echocardiographic evaluation is showed in Fig. 1.

\section{TAVI procedure}

TAVI was performed using a standard trans-femoral or -subclavian technique. Both balloon-expandable and selfexpandable valves were used for this purpose. Patients with stable CAD and significant stenosis $(>70 \%)$ underwent revascularization with percutaneous angioplasty and drug-eluting stent implantation at least one month before the procedure. A self-expandable valve was implanted in 40 patients $(60 \%)$, while a balloon-expandable valve was implanted in 28 patients $(40 \%)$.

\section{Statistical analysis}

Statistical analysis was performed using SPSS software v.23. Continuous variables were reported as mean \pm standard deviation, and were compared using Student's t test or the Mann-Whitney rank sum test for unpaired and paired comparisons, as appropriate. Categorical variables were reported as percentages of individuals. The $\chi 2$ test or Fisher's exact test were used to compare qualitative variables. Differences were considered statistically significant when $\mathrm{p}<0.05$.

\section{Results}

No significant differences in baseline clinical characteristics were found between the two groups, including the presence of stable CAD, as showed in Table 1. In Table 2 are reported standard echocardiographic characteristics, while Table 3; Fig. 2 show ST-E analysis before and after TAVI. Patients with $\mathrm{cLVH}$ were more likely to have smaller LV end-diastolic volume, although no significant (respectively, LVEDV $/ \mathrm{i} 55 \pm 15 \mathrm{ml} / \mathrm{m}^{2}$ vs. $62 \pm 14, \mathrm{p}=0.066$ ) and higher values of LS at baseline in all layers (respectively EndoLS was $-13.2 \pm 2$ vs. $-11.1 \pm 3 \%, p=0.041$; Epi-LS was $-11.8 \pm 1.8$ vs. $-9.9 \pm 3 \%, p=0.043$; Transmural-LS was $-12.3 \pm 1.8$ vs. $--0.49 \pm 3.3 \%, p=0.02$ ). Valvulo-arterial impedance (Zva) was similar in the two groups. After TAVI, a significant improvement in Endo-LS was observed only in cLVH group $(-13 \pm 2$ vs. $-4 \pm 2, p=0.011)$. No significant improvements were recorded in eLVH patients after TAVI. Standard echocardiographic parameters didn't show any significant amelioration immediately after TAVI. No differences in type of valve implanted were found in the two groups. 

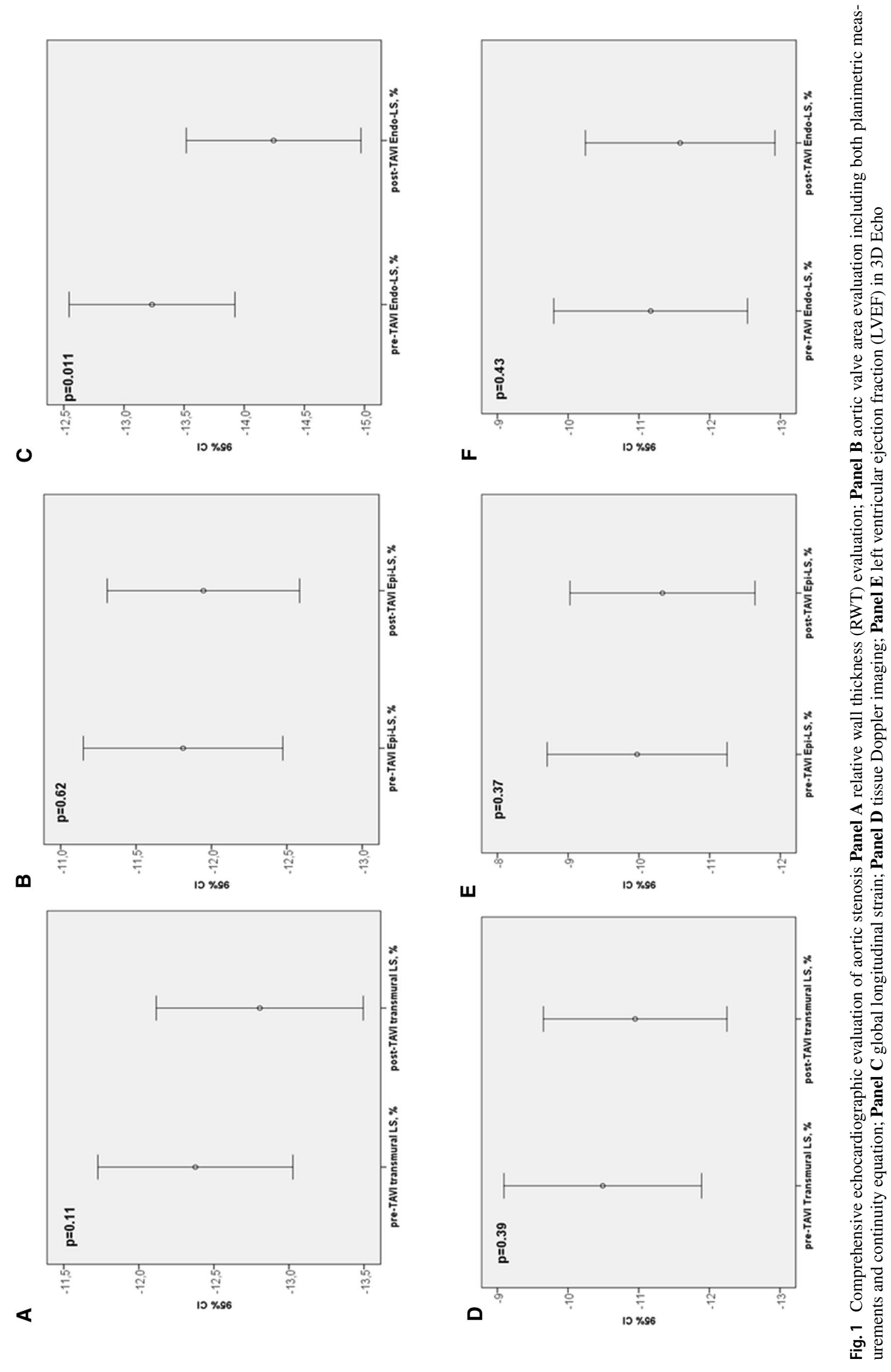

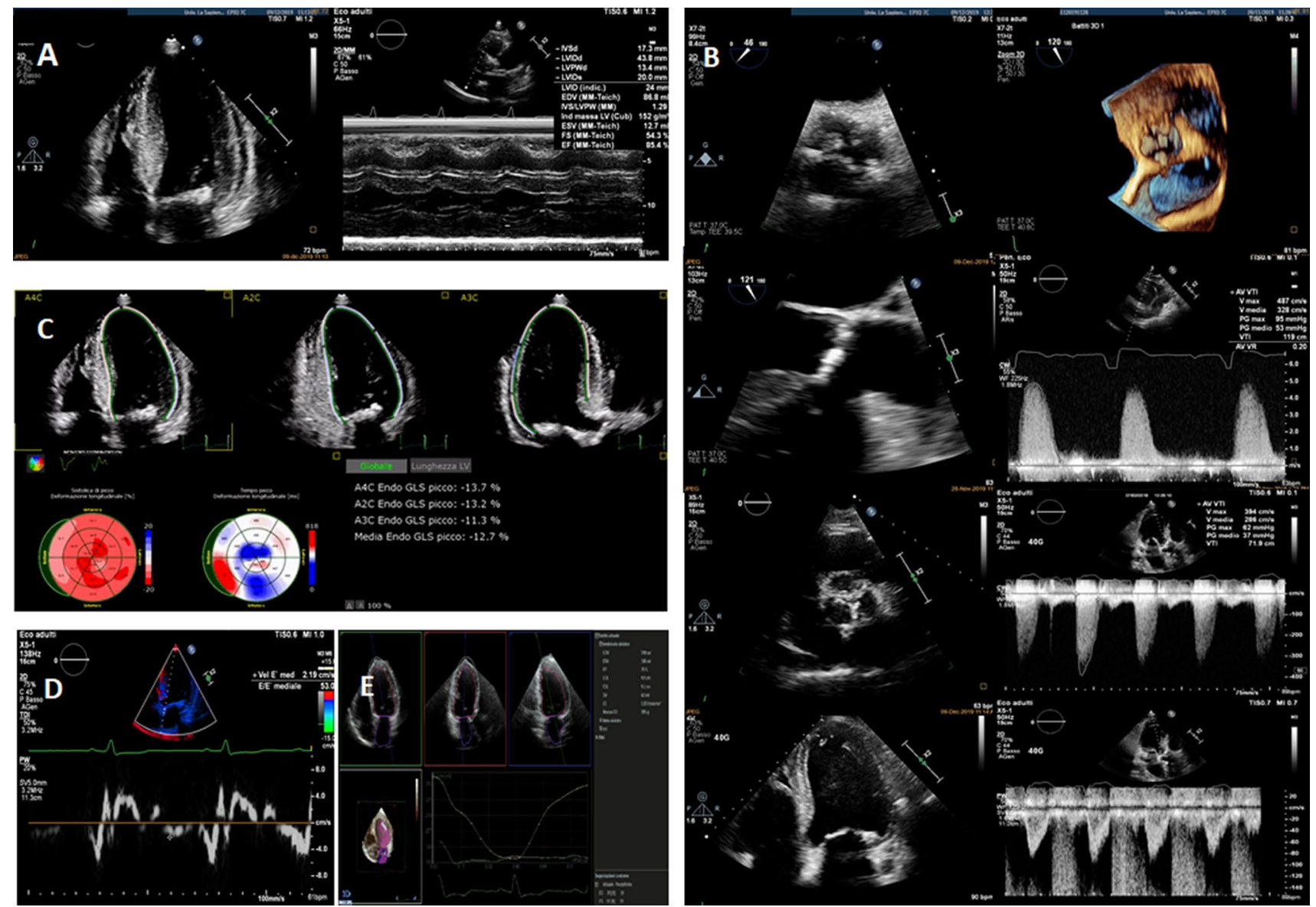

Fig. 2 The top row shows differences in transmural (a), epicardial (b) and subendocardial (c) strain before and after TAVI, in cLVH patients. The bottom row shows differences in transmural (e), epicardial (f) and subendocardial (g) strain before and after TAVI, in eLVH patients

Table 1 Clinical and demographic characteristics

\begin{tabular}{llll}
\hline Parameter & cLVH 46 pts (68\%) & eLVH 22 pts (32\%) & p value \\
\hline Age, y.o. & $82 \pm 3$ & $83 \pm 5$ & 0.21 \\
Male sex, n (\%) & $14(30)$ & $11(50)$ & 0.31 \\
STS Score & $3 \pm 0.8$ & $4 \pm 1.1$ & 0.08 \\
EuroScore II & $4.7 \pm 2$ & $4.8 \pm 3$ & 0.93 \\
Hypertension, n (\%) & $37(80)$ & $18(81)$ & 0.44 \\
Diabetes, n (\%) & $11(25)$ & $5(25)$ & 0.48 \\
Dyslipidemia, n (\%) & $23(50)$ & $14(63)$ & 0.68 \\
CRF (stage III-IV), n (\%) & $5(10)$ & $2(10)$ & 0.99 \\
CAD, n (\%) & $18(40)$ & $9(40)$ & 0.22 \\
\hline
\end{tabular}

$C A D$ coronary artery disease, $C R F$ chronic renal failure

\section{Discussion}

The present study showed (a) the presence of less impaired LS values at baseline, in all layers, in patients with cLVH than in patients with eLVH; (b) a significant improvement of Endo-LS early after TAVI, only in cLVH.

LVH in AS occurs as a response to pressure overload and cLVH pattern is more frequently observed than eLVH, particularly when valve regurgitation and LV systolic dysfunction are excluded [11].The presence of excessive LVH was considered as a negative prognostic marker in patients 
Table 2 Echocardiographic characteristics in patients with aortic stenosis presenting different LV geometry

\begin{tabular}{|c|c|c|c|}
\hline Parameters & $\begin{array}{l}\text { cLVH } \\
46 \text { pts }(68 \%)\end{array}$ & $\begin{array}{l}\text { eLVH } \\
22 \text { pts (32\%) }\end{array}$ & $\mathrm{p}$ \\
\hline \multicolumn{4}{|l|}{ Before TAVI } \\
\hline Ao Vmax, m/sec & $4.29 \pm 0.7$ & $4.12 \pm 0.5$ & 0.56 \\
\hline Ao MPG, mmHg & $49 \pm 15$ & $46 \pm 10$ & 0.41 \\
\hline Ao PPG,mmHg & $70 \pm 22$ & $70 \pm 17$ & 0.25 \\
\hline AVA, $\mathrm{cm}^{2}$ & $0.8 \pm 0.18$ & $0.7 \pm 0.3$ & 0.60 \\
\hline AVA $/ \mathrm{i}, \mathrm{cm}^{2} / \mathrm{m}^{2}$ & $0.39 \pm 0.12$ & $0.38 \pm 0.07$ & 0.63 \\
\hline $\mathrm{Zva}, \mathrm{mmHg} * \mathrm{~m}^{2} / \mathrm{ml}$ & $4.1 \pm 1.3$ & $4.4 \pm 1.1$ & 0.46 \\
\hline PLFLG, n (\%) & $8(17)$ & $2(10)$ & 0.23 \\
\hline $\mathrm{LVEDV} / \mathrm{i}, \mathrm{ml} / \mathrm{m}^{2}$ & $55 \pm 15$ & $62 \pm 14$ & 0.06 \\
\hline $\mathrm{LVESV} / \mathrm{i}, \mathrm{ml} / \mathrm{m}^{2}$ & $24 \pm 6$ & $28 \pm 9$ & 0.14 \\
\hline LVEF, \% & $55 \pm 5$ & $56 \pm 6$ & 0.99 \\
\hline $\mathrm{SV} / \mathrm{i}, \mathrm{ml} / \mathrm{m}^{2}$ & $41 \pm 11$ & $41 \pm 10$ & 0.82 \\
\hline LV mass, $\mathrm{g} / \mathrm{m}^{2}$ & $158 \pm 35$ & $166 \pm 54$ & 0.15 \\
\hline $\mathrm{LAVi}, \mathrm{ml} / \mathrm{m}^{2}$ & $48 \pm 19$ & $55 \pm 21$ & 0.15 \\
\hline $\mathrm{E} / \mathrm{A}$ & $0.79 \pm 0.3$ & $0.93 \pm 0.5$ & 0.25 \\
\hline $\mathrm{E} / \mathrm{e}^{\prime}$ & $12 \pm 8$ & $15 \pm 4$ & 0.08 \\
\hline TTG, mmHg & $29 \pm 11$ & $32 \pm 10$ & 0.45 \\
\hline TAPSE, mm & $22 \pm 3$ & $20 \pm 2$ & 0.1 \\
\hline RV LS, \% & $-18 \pm 5$ & $-16 \pm 4$ & 0.36 \\
\hline \multicolumn{4}{|l|}{ After TAVI } \\
\hline LVEDV $/ \mathrm{i}, \mathrm{ml} / \mathrm{m}^{2}$ & $56 \pm 14$ & $63 \pm 13$ & 0.07 \\
\hline $\mathrm{LVESV} / \mathrm{i}, \mathrm{ml} / \mathrm{m}^{2}$ & $23 \pm 5$ & $27 \pm 8$ & 0.19 \\
\hline LVEF, \% & $55 \pm 6$ & $56 \pm 7$ & 0.99 \\
\hline $\mathrm{LAVi}, \mathrm{ml} / \mathrm{m}^{2}$ & $47 \pm 18$ & $56 \pm 22$ & 0.25 \\
\hline E/A & $0.8 \pm 0.2$ & $0.9 \pm 0.6$ & 0.32 \\
\hline $\mathrm{E} / \mathrm{e}^{\prime}$ & $11 \pm 9$ & $16 \pm 4$ & 0.09 \\
\hline TTG, mmHg & $24 \pm 11$ & $34 \pm 11$ & 0.48 \\
\hline TAPSE, mm & $21 \pm 4$ & $20 \pm 3$ & 0.99 \\
\hline Self-expandable valve, $\mathrm{n}(\%)$ & $28(60 \%)$ & $12(54 \%)$ & 0.8 \\
\hline Balloon-expandable valve, n (\%) & $18(40 \%)$ & $10(46 \%)$ & 0.8 \\
\hline
\end{tabular}

Ao aortic, AVA aortic valve area, $c L V H$ concentric left ventricular hypertrophy, $e L V H$ eccentric left ventricular hypertrophy, $L A V$ left atrial volume, $L V E D V$ left ventricular end-diastolic volume, $L V E S V$ left ventricular end-systolic volume, $L S$ longitudinal strain, $L V E F$ left ventricular ejection fraction, $M P G$ mean pressure gradient, $P L F L G$ paradoxical low flow low-gradient, $P P G$ peak pressure gradient, $R V$ right ventricle, TAPSE tricuspidal annular systolic escursion, TTG trans-tricuspidal gradient, ZVAValvulo-arterial impedance

managed conservatively. Few data are available on the prognostic value of eLVH in this subset of patients, due to the less common presentation [11]. However, since the natural history of AS leads to heart failure (HF), the transition point from the compensatory LVH to decompensation and $\mathrm{HF}$ is known to be related with fibrosis and myocardial apoptosis and occurs when the LV fails to counterbalance an increased pressure afterload and is no longer able to maintain forward flow through the stenotic valve. In this stage of the disease, cLVH may shift to eLVH [12].
Table 3 Multilayer LV strain analysis before and after TAVI

\begin{tabular}{llll}
\hline Parameters & cLVH & eLVH & $p$ \\
& 46 pts $(68 \%)$ & 22 pts (32\%) & \\
\hline Before TAVI & & & \\
Transmural-LS, \% & $-12.3 \pm 1.8$ & $-10.49 \pm 3.3$ & $\mathbf{0 . 0 2}$ \\
Epi-LS, \% & $-11.8 \pm 1.8$ & $-9.9 \pm 3$ & $\mathbf{0 . 0 4 3}$ \\
Endo-LS, \% & $-13 \pm 2$ & $-11 \pm 3.2$ & $\mathbf{0 . 0 4 1}$ \\
LS gradient, \% & $-1.4 \pm 0.7$ & $-1.2 \pm 0.6$ & 0.08 \\
After TAVI & & & \\
Transmural-LS, \% & $-12.8 \pm 1.9$ & $-10.8 \pm 3$ & $\mathbf{0 . 0 4 5}$ \\
Epi-LS, \% & $-11.9 \pm 1.9$ & $-10.3 \pm 3$ & $\mathbf{0 . 4 8}$ \\
Endo-LS, \% & $-14 \pm 2$ & $-11.5 \pm 3.17$ & $\mathbf{0 . 0 0 3}$ \\
LS gradient, \% & $1.6 \pm 0.7$ & $1.12 \pm 0.7$ & 0.06
\end{tabular}

Bold values represent statistically significant values

Endo endocardial, Ep epicardial, $L S$ longitudinal strain, other abbreviations as above

Accordingly, LS is known to be impaired in AS. It was previously demonstrated that the mechanisms involved in the alteration of LS in AS are (a) the development of LVH and (b) LV fibrosis, which is related with both increased afterload and concomitant CAD [13]. Layer-specific strain pattern is already known to be altered in $\mathrm{LVH}$ with different etiologies [14].

Previous studies already explored LS behavior in different myocardial layers after TAVI [15, 16]. Shiino et al. [15] demonstrated a significant LS improvement in all myocardial layers, which was more prominent in sub-endocardial layer, as expected. The same behaviour was observed by Kim et al. [16] who found that LS improvement was greater in patients with higher grade of LVH. In line with these results, our data confirmed that after LV unloading only Endo-LS shows a significant early improvement, but exclusively in one specific subset of patients (cLVH). Our data demonstrated a significant worse baseline LS pattern in eLVH compared to cLVH in all myocardial layers, further supporting a more favorable setting in cLVH. Indeed, eLVH is known to be more frequently associated with further development of heart failure and poor LV adaptation to stress than cLVH [17], and the lack of early Endo-LS recovery in this subset could be a marker of poor prognosis. Our results have to be confirmed in larger longitudinal studies and have to be compared with clinical and echocardiographic follow-up. These preliminary observations have potential implications: advanced valve disease with more severe and eccentric LVH can have less benefits form LV unloading; LV geometry should be taken in account in the decision making process. 


\section{Limitations}

The main limitation of the present study is the small sample size that could mask other differences between groups. Follow-up data are not provided, since the study is not powered for MACCE. Finally, there is no control group for comparison.

\section{Conclusions}

Our results demonstrated that patients with severe aortic stenosis and cLVH geometry had a better improvement after TAVI, described by multilayer LS analysis, compared to eLVH. This result highlights the possible role of LV geometry to improve the selection of the stage of valve disease, identifying who could have less benefit from the reduced afterload after aortic replacement.

Funding Open access funding provided by Università degli Studi di Roma La Sapienza within the CRUI-CARE Agreement.

\section{Compliance with ethical standards}

Conflict of interest The authors declare that thye have no Conflict of interest.

Open Access This article is licensed under a Creative Commons Attribution 4.0 International License, which permits use, sharing, adaptation, distribution and reproduction in any medium or format, as long as you give appropriate credit to the original author(s) and the source, provide a link to the Creative Commons licence, and indicate if changes were made. The images or other third party material in this article are included in the article's Creative Commons licence, unless indicated otherwise in a credit line to the material. If material is not included in the article's Creative Commons licence and your intended use is not permitted by statutory regulation or exceeds the permitted use, you will need to obtain permission directly from the copyright holder. To view a copy of this licence, visit http://creativecommons.org/licenses/by/4.0/.

\section{References}

1. Rader F, Sachdev E, Arsanjani R, Siegel RJ (2015 Apr) Left ventricular hypertrophy in valvular aortic stenosis: mechanisms and clinical implications. Am J Med 128(4):344-352

2. Infusino F, Calcagno S, Cimino S, Pucci M, Salvi N, Maestrini V et al (2020) Left ventricular wall stress is associated with myocardial functional recovery in patients with severe aortic stenosis and systolic dysfunction undergoing transcatheter aortic valve replacement. J Cardiovasc Med (Hagerstown). https://doi.org/10.2459/ JCM.0000000000001069

3. Davin L, Nchimi A, Ilardi F, Dulgheru R, Marchetta S, Gach O et al (2019) Epicardial adipose tissue and myocardial fibrosis in aortic stenosis relationship with symptoms and outcomes: a study using cardiac magnetic resonance imaging. JACC Cardiovasc Imaging 12(1):213-214

4. Gaasch WH, Zile MR (2011) Left ventricular structural remodeling in health and disease: with special emphasis on volume, mass and geometry. J Am Coll Cardiol 58(17):1733-1740

5. Treibel TA, Badiani S, Lloyd G, Moon JC (2019) Multimodality imaging markers of adverse myocardial remodeling in aortic stenosis. JACC Cardiovasc Imaging 12(8 Pt 1):1532-1548

6. Baumgartner H, Falk V, Bax JJ, De Bonis M, Hamm C, Holm PJ, ESC Scientific Document Group et al (2017) ESC/EACTS guidelines for the management of valvular heart disease. Eur Heart $\mathbf{J}$ 38(36):2739-2791

7. Hita A, Baratta S, Vaccarino G, Navia J, Oleno D, Telayna JM et al (2015) Severe aortic stenosis with preserved ejection fraction and evidence of impairment in structure, myocardial strain and ventricular function: a new contribution to clinical decision making. Cardiol J 22(6):613-621

8. Alcidi GM, Esposito R, Evola V, Santoro C, Lembo M, Sorrentino $R$ et al (2018) Normal reference values of multilayer longitudinal strain according to age decades in a healthy population: a single-centre experience. Eur Heart J Cardiovasc Imaging 19(12):1390-1396

9. Ilardi F, Marchetta S, Martinez C, Sprynger M, Ancion A, Manganaro R et al (2020) Impact of aortic stenosis on layer-specific longitudinal strain: relationship with symptoms and outcome. Eur Heart J Card Imaging. https://doi.org/10.1093/ehjci/jez215

10. Merdler I, Loewenstein I, Hochstadt A, Morgan S, Schwarzbard S, Sadeh B et al (2020) Effectiveness and safety of transcatheter aortic valve implantation in patients with aortic stenosis and variable ejection fractions $(<40 \%, 40 \%-49 \%$, and $>50 \%)$. Am J Cardiol 15(4):583-588

11. Debry N, Maréchaux S, Rusinaru D, Peltier M, Messika-Zeitoun D, Menet A et al (2017) Prognostic significance of left ventricular concentric remodelling in patients with aortic stenosis. Arch Cardiovasc Dis 110(1):26-34

12. Dweck MR, Boon NA, Newby DE (2012 Nov) Calcific aortic stenosis: a disease of the valve and the myocardium. J Am Coll Cardiol 6(19):1854-1863 60(

13. Reant P, Metras A, Detaille D, Reynaud A, Diolez P, JaspardVinassa B et al (2016) Impact of afterload increase on left ventricular myocardial deformation indices. J Am Soc Echocardiogr 29(12):1217-28

14. Huang M, Lee WH, Tsai HR, Liu YW, Liu PY, Tsai WC (2019) Value of layer-specific strain distribution patterns in hypertrophied myocardium from different etiologies. Int J Cardiol 281:69-75

15. Shiino K, Yamada A, Scalia GM, Putrino A, Chamberlain R, Poon $\mathrm{K}$ et al (2019) Early changes of myocardial function after transcatheter aortic valve implantation using multilayer strain speckle tracking echocardiography. Am J Cardiol 123 (6)(3):956-960

16. Kim HJ, Lee SP, Park CS, Park JB, Kim YJ, Kim HK, Sohn DW (2016) Different responses of the myocardial contractility by layer following acute pressure unloading in severe aortic stenosis patients. In J Cardiovasc Imaging 32:247-259

17. Rozenbaum Z, Finkelstein A, Zhitomirsky S, Topilsky Y, Halkin A, Banai S et al (2020 Feb) Impact of preprocedural left ventricle hypertrophy and geometrical patterns on mortality following TAVR. Am Heart J 220:184-191

Publisher's Note Springer Nature remains neutral with regard to jurisdictional claims in published maps and institutional affiliations. 